\title{
ESTANDARIZACIÓN DEL TEST DE APTITUDES ESCOLARES T.A.E. NIVELES 1 Y 2
}

César Ruiz Alva

El presente es el resultado de la estandarización del Test Aptitudes Escolares TAE niveles 1 y 2, para lo cual se aplicó a 5980 estudiantes del 3ro a 6to. De primaria y de 1ro. a 5to.de secundaria cuyas edades fluctuaron entre los 8 a 16 años, del total muestral, 2580 correspondieron a la primaria y 3400 al nivel secundario. La confiabilidad del test por el método de las mitades para el TAE 1 arrojó coeficientes de 0,90 como mínimo hasta 0,93 como máximo y para el TAE 2 de 0,89 como mínimo hasta 0,92 .

La validez se efectuó mediante el método concurrente correlacionando los resultados con el rendimiento académico en las asignaturas de matemática, lenguaje y rendimiento en general, asimismo, se correlacionó con el Test Factor "G" forma 2 adaptada por la doctora Nelly Ugarriza.

Las normas se elaboraron por edad cronológica en la escala coeficiente intelectual y también por grados escolares.

PALABRAS CLAVE: aptitud escolar, con fiabilidad, validez, inteligencia. normalización.

The present is the result of the standardization of the Test School Aptitudes T AE levels 1 and 2, for which I apply 5980 students of the 3ro. to 6 to. of primary and of 1ro. To 5to. of secondary whose ages fluctuated among the 8 to 16 years, of the total one muestral, 2580 they corresponds to the primary and 3400 at the secondary level. The dependability of the test by the method of the halves for the TAE 1 I throw coefficients of 0,90 ace minimum to 0,93 at most and for the T AE 2 of 0,89 ace minimum to 0,92 .

The validity was performed by means of the concurrent method correlating the results with the academic performance in the subjects of mathematics, language and performance in general, likewise, I am correlated with the test factor $\mathrm{g}$ forms 2 adapted by the grants a doctorate Nelly Ugarriza. The norms elaborated for chronology age in the intellectual scale coefficient and also by school degrees.

KEY WORDS: school aptitude, dependability, validity, intelligence, normalization. 


\section{INTRODUCCIÓN}

Para quienes utilizamos los tests psicológicos dentro de nuestra labor diagnóstica, un requisito importante es, que los instrumentos se adecuen a la realidad en la cual trabajamos. Supone esto que la estandarización del test, como una de las mayores exigencias, deba ser cumplida a cabalidad. Al final se asegura con esto que los resultados se analicen con objetividad y ayude a una toma de decisiones con un alto índice de certidumbre.

Es por eso que, nos interesó estandarizar un test psicológico escogiendo el TEST DE APTITUDES ESCOLARES, (Niveles 1-2) ya que servirá para que los Psicólogos educacionales principalmente cuenten con una herramienta que ayude a evaluar las aptitudes escolares de los alumnos cuyas edades estén entre los 8 y los 16 años.

En este documento se condensan los aspectos fundamentales de la investigación llevadas a cabo dentro del marco de proyectos presentados y aprobados durante 1994 por el Instituto de Investigaciones Psicológicas, cuyo financiamiento ha hecho posible cubrir los costos que demanda trabajos de esta naturaleza. Así, señalamos que todos los procedimientos técnicos empleados en el estudio referentes a la justificación estadística, aplicación, valoración e interpretación de resultados, incluidos los Baremos que se elaboraron con la amplia muestra que se utilizó.

Deseo expresar mi gratitud a todas aquellas personas que colaboraron en este proyecto, en especial a mis colegas los profesores Abel Cuzcano Zapata y Pablo Félix Castañeda. También mi reconocimiento a la dedicación y entrega a este trabajo de parte de la Psicóloga Eva Guerra Turín, mi apoyo permanente y brazo derecho no solo en la recolección de datos sino principalmente en la organización, revisión de manuscritos y tipeo. Finalmente debo dar gracias a mis alumnos de la práctica pre profesional de Psicología Educacional tanto de la Universidad de San Marcos como de la Universidad Inca Gracilazo de la Vega por haber sido los peones en este trabajo, recogiendo muestras de todos los rincones de Lima a donde fueron enviados, mostrando siempre un alto espíritu de colaboración y siempre dispuestos a aprender día a día los pasos para llevar a cabo una estandarización. Esta tarea que se logró con los aportes de cada uno de mis colaboradores culminó con la elaboración del Manual que se presenta al interior de estas páginas, el cual servirá para el uso de esta prueba psicológica.

\section{CARACTERÍSTICAS GENERALES DEL T.A.E. NIVELES 1 Y 2}

\subsection{FICHA TÉCNICA:}

Nombre Original: "SRA Tests of Educational Ability"

Autores: L.L. Thurstone y Thelma Thurstone

Nombre de la adaptación en español: TEA "Test de Aptitudes Escolares". Niveles 1 y 2.

Adaptación y Normalización en Perú: César Ruiz Alva.

Institución: Instituto de Investigaciones Psicológicas de la Universidad Nacional Mayor de San Marcos, Lima, 1995.

Administración: Individual y Colectiva.

Duración aproximada:

Nivel 1 (30 minutos)

Nivel 2 (40 minutos)

Niveles de aplicación:

Nivel 1 (3ro a 6to grado de Primaria) 
Nivel 2 (1ro a 5to grado de Secundaria)

Significado del test: Evalúa las aptitudes fundamentales que se exigen en las tareas escolares.

Baremación: Tablas de C.I. (Coeficiente Intelectual) para las diferentes edades de

aplicación y Percentiles para los diversas grados escolares.

Materiales: Cuadernillo, Hoja de respuestas, Plantilla par nivel.

\subsection{MARCO TEÓRICO}

\subsubsection{Las Aptitudes}

Existen diversas enfoques acerca del concepto de Aptitud, presentaremos a continuación una síntesis de las más frecuentes:

a). Unos autores consideran que la aptitud implica Diferencias Individuales ya que son estas las que determinan los elementos explicativos de las variaciones a diferencias entre las personas, dado que, los individuos las poseen en diferentes grados de desarrollo. Estas diferencias pueden depender de factores hereditarias que representan las potencialidades funcionales, pero también dependen de factores ambientales que representan las experiencias vitales del sujeto o de la confluencia de ambos tipos de factores sean hereditarias o ambientales.

b). Otros le ponen énfasis a un índice de Rendimiento pues consideran que si se quiere evaluar las aptitudes con pruebas psicológicas el resultado de dicha medida será el nivel de rendimiento alcanzado y

no la medida de un factor aptitudinal sea hereditaria o ambiental por sí sola. De esto resulta que, vista así las aptitudes son capacidades que permiten a las individuos adquirir eficiencia y rapidez en la ejecución de las actividades.

c). Aún hay quienes buscan establecer diferencias conceptuales entre aptitud y capacidad y ponen énfasis en que la aptitud se asocia a particularidades anatómicas que forman las diferencias innatas de las personas y que permiten el desarrollo de las capacidades, pero éstas nunca las predeterminan, ya que las capacidades se desarrollan según se desenvuelva la vida del sujeto. Así, toda capacidad, es condición para realizar con éxito. determinado tipo de actividad. Aptitud entonces se asocia a potencialidad innata y capacidad para la disposición adquirida.

d). También hay autores que centran su enfoque en las realizaciones del individuo, es decir, plantean que si se quiere diferenciar entre unas y otras, se debe conservar la noción de realización o grado de éxito en la ejecución de una tarea. La aptitud es algo que se manifiesta día a día, produce sus efectos a través de la realización, y es a partir del nivel de ésta última como se puede predecir un comportamiento subsiguiente. Es la aptitud la capacidad para hacer algo y sus características pueden ser experimentalmente verificadas.

e). Finalmente, mencionaremos los enfoques sobre aptitud que se asocian al factor aprendizaje, definiéndola como la condición a serie de características consideradas como síntomas de la capacidad de un individuo para adquirir, con un entrenamiento. y; adecuado algún conocimiento, habilidad o serie de razones como la capacidad para aprender idiomas, componer música, etc. En esta línea la aptitud es vista como la capacidad para adquirir cierta variedad de conductas o habilidades, de tal modo que el factor común sea la capacidad para aprender y no el tipo de conductas aprendidas.

Los autores de la Prueba Test de Aptitudes Escolares coinciden con esta última posición ya que consideran a la aptitud como la capacidad para aprender, para adquirir habilidades y conocimientos impartidos en colegio, $y$, sobre la base de estas capacidades poder estimar una predicción del éxito o fracaso de los alumnos en el proceso enseñanza-aprendizaje. 
Compartimos, en este trabajo, la concepción de que aptitud escolar es aquella condición individual o diferenciadora que posibilita al sujeto la adquisición de aprendizajes, que se expresa en el manejo dé representaciones verbales, espaciales, y en la ejecución de cálculos numéricos, todas ellas predominantes en los programas curriculares que se siguen en los centros educativos.

1.2.2. Desarrollo de las Aptitudes Asumiendo la posición de Fingerman (1971) las aptitudes pasan por tres etapas de desarrollo:

\section{Etapa Progresiva}

La aptitud aparece en forma espontánea y se va desarrollando en forma gradual hasta llegar a la madurez. No todas las aptitudes se desarrollan a la vez ya que el ritmo de desarrollo de cada aptitud particular puede ser diferente en cada individuo. Además, este ritmo de desarrollo se ve afectado por la influencia de factores individuales y del medio ambiente.

\section{Etapa Estática}

La aptitud que alcanzó cierto grado de desarrollo, se mantiene durante un tiempo relativamente largo a un mismo nivel.

\section{Etapa Regresiva}

La aptitud a causa de ciertas circunstancias, como por ejemplo, la edad, comienza a debilitarse y deformarse.

\subsubsection{Clasificación de las Aptitudes}

Siguiendo a Fingerman las aptitudes de acuerdo a la naturaleza de sus funciones pueden ser agrupadas en cuatro categorías:

\section{Aptitudes Sensoriales}

Son las disposiciones que nos permiten distinguir colores, percibir formas, diferencias, ruidos apenas perceptibles, reconocer y diferenciar olores, sabores, etc.

\section{Aptitudes Motrices}

Son las disposiciones para realizar movimientos ya sean estos firmes, rápidos, lentos o de precisión.

\section{Aptitudes Afectivas}

Referidas a las disposiciones para sentir lo bello o lo feo, lo agradable o desagradable. La aptitud estética puede referirse ya sea al dibujo, pintura, sentido de proporciones, simetría, etc.

\section{Aptitudes Intelectuales}

Referidas a disposiciones de carácter enteramente psicológico, como la memoria, la atención y diversos componentes intelectuales y, en los cuales va a intervenir el sistema nervioso central.

\subsubsection{Enfoque Multifactorial de la Inteligencia}

Tradicionalmente en Psicología y también en Educación cuando habla de Inteligencia y su medición, se hace referencia a enfoques cuantitativos ya las pruebas de aptitud 
psicológica en las que. se basa. Existen dos enfoques cuantitativos bastante diferentes. uno es el unitario. que considera a la inteligencia en términos holísticos como una función general. El otro es el factorial que interpreta a la inteligencia de una forma más atomista, en el sentido de que está integrada por un número de componentes distintos y no muy relacionados. cada uno de los cuales son susceptibles de medición individual. Otra definición para el enfoque cuantitativo, tal vez de mayor significancia descriptiva, es el enfoque analítico psicométricos.

\section{Sobre la Naturaleza de la Inteligencia}

La capacidad de comportarse con inteligencia es una de las más valiosas aptitudes del ser humano. Constituye un atributo gracias al cual su conducta se vuelve distintivamente humana. Le permite aprender, razonar, aprovechar sus experiencias del pasado, predecir el futuro, manipular su medio ambiente y trascender con su pensamiento las barreras del tiempo y del espacio.

Algunos autores definen la inteligencia como:

a) Habilidad para aprender y para manipular objetos.

b) Flexibilidad y capacidad del individuo para ajustarse al medio.

Junto a ellos hay autores para quienes la inteligencia debe también ser evaluada en términos de afecto, valores y actitudes y no sólo haciendo referencia a las habilidades puramente cognoscitivas.

Evidentemente la inteligencia no es una entidad que se pueda aislar y examinar en cualquier sentido físico. Las mediciones de la inteligencia son indirectas y tan sólo examinan la habilidad de un individuo para desempeñar las conductas que se reconocen como representativas de la inteligencia y que permiten predecir el probable nive1 intelectual de la conducta dc una persona.

\section{La Teoría de Thurstone sobre Inteligencia}

La Teoría Multifactorial propuesta por Thurstone, postula que existen un número de factores distintos en la conducta cognoscitiva teniendo todas ellas un factor común, similar al factor $G$ de Spearman. Thurstone, en un principio separó ocho factores principales (Habilidades Mentales Primarias) de la siguiente manera: Verbal (V),velocidad perceptual $(\mathrm{P})$, numérico $(\mathrm{N})$ razonamiento inductivo $(\mathrm{R})$, memoria mecánica $(\mathrm{M})$, razonamiento deductivo (D), fluidez verbal (F) y espacial (E). Mas tarde, el autor modifica sus factores pero en general estos han permanecido relativamente estables y el modelo teórico ha servido para diseñar pruebas como el Test de Aptitudes escolares que explora fundamentalmente factores de tipo verbal, numérico y de razonamiento como medida de la inteligencia de los estudiantes.

\subsection{DESCRIPCIÓN,GENERAL DE LOS NIVELES 1 Y 2}

El Test de Aptitudes Escolares fue preparado para medir tres dimensiones aptitudinales o factores mentales siguientes:

-Verbal (V)

- Razonamiento (R)

- Cálculo (C)

Al combinar estos factores se obtiene una puntuación total que se transforma en un Coeficiente Intelectual (C.I.) Es posible obtener Puntuaciones Percentiles por grados escolares.

A continuación presentamos un cuadro que esquematiza las pruebas, número de ítems, y 
descripción de lo que cada una mide en el TAE:

\section{Cuadro 1}

Descripción de los Niveles del Test de Aptitudes Escolares

\begin{tabular}{|l|c|c|l|}
\hline \multicolumn{1}{|c|}{ Prueba } & $\begin{array}{c}\mathbf{N}^{\mathbf{0}} \text { de } \\
\text { Items }\end{array}$ & Nivel & \multicolumn{1}{|c|}{ Significado de lo que mide } \\
\hline Dibujos & 15 & ---- & Identificación verbal de Fig. \\
Palabras Dif. & 15 & 20 & Razonamiento verbal \\
Vocabulario & 20 & 30 & Comprensión verbal /sinónimos \\
\hline Razonamiento & 27 & --- & Razonamiento con figuras \\
Series de No & --- & 15 & Razonamiento con números \\
Series letras & --- & 15 & Razonamiento con letras \\
\hline Cálculo & 55 & 30 & Comprensión numérica \\
\hline Total & 132 & 140 & Aptitudes fundamentales que se exigen \\
\hline
\end{tabular}

\subsection{UTILIDAD DEL INSTRUMENTO T.A.E}

El test de Aptitudes Escolares. en la medida en que busca evaluar aquellas capacidades exigidas para desenvolverse adecuadamente en el; campo educativo. puede ser de gran utilidad para el Psicólogo Educacional. como para los educadores o tutores a fin de que estimen sobre las aptitudes alcanzadas por los alumnos, los niveles de aprovechamiento escolar que debe exigirse en el presente; asimismo, juzgar su proyección para el desempeño futuro en la educación o carrera a seguir.

Sobre. todo la Puntuación total constituye un elemento valioso para el análisis pues se demuestra una alta correlación tanto con tests de inteligencia como con rendimientos académicos para los diversos grados escolares. Consecuentemente, esa puntuaciones total servirá para;

a). Identificar aquellos alumnos con una alta dotación en sus aptitudes escolares (Percentiles 80 o superiores).

b).Diferenciar a los escolares con baja dotación aptitudinal para quienes es necesario que sigan cursos de recuperación o Complementación

(Percentiles 25 o menos).

e). Obtener el C.I. promedio de cada grupo, sección. grado.

d). Elaborar baremos especificas ,para cada centro escolar, así como establecer correlaciones con otras pruebas o calificaciones que se obtengan en el centro.

e). Agrupar a los alumnos dentro de cada clase según su dotación baja, media o alta. a fin de beneficiarlos con una enseñanza más efectiva.

f). Identificar a los alumnos con una pobre motivación, si los resultados de las pruebas son altos pero su rendimiento escolar es bajo (probablemente atiende a las tareas escolares con poca disposición y una ejecución inferior a lo que podría esperarse por sus aptitudes potenciales). Estos alumnos rendirían mejor si se les da una atención especial.

g). Tener un marco de referencia para entrevistas con alumnos o padres respecto al nivel aptitudinal para Programas de Orientación Vocacional. 


\section{ADAPTACION PERUANA DEL T.A.E.}

Esta adaptación y Normalización se ha llevado a cabo a partir de la Edición 1972 del T AE. En el proceso se han seguido fases similares a la adaptación española trabajando en paralelo la recolección de datos de centros primarios y secundarios por la naturaleza de las escuelas y colegios en nuestro medio.

La finalidad principal del trabajo ha tenido como metas importantes, adecuar el test para su uso en nuestra realidad y, establecer las normas que permitan comparar los rendimientos de nuestra población, de un modo similar a cómo se procedió en la versión original.

\section{Etapas del Proceso}

1. Revisión de la bibliografía sobre el tema y antecedentes sobre estudios con el TAE.

2. Revisión y adecuación del material del tests (adaptarla a la realidad nacional pero cuidando conservar la estructura y tipo de formulación originales.

3. Redacción de un Manual de Instrucciones. Uso de Protocolos y Tests y preparación de grupo evaluador (formado por Profesores de Apoyo y de Internistas del área Educacional de la Facultad de Psicología de la Universidad Nacional Mayor de San Marcos).

4. Reajuste de materiales, Elaboración de Manual definitivo, preparación de pruebas y protocolos finales.

5. Coordinaciones y estudios de la composición de grupos normativos para cada nivel. Recolección de datos previos.

6. Toma de las pruebas. Aplicaciones colectivas a grupos hasta de 25 alumnos En el T AE 1 se trabajó en grupos de 5 a 15 niños.

7. Calificación, puntuación y valoración de resultados para obtener los estadísticos de la Normalización que-se buscaba.

8. Elaboración final del Manual, Cuadros, Normas, Validez, Con fiabilidad y el Reporte de Investigación.

\section{INFORMACIÓN ESTADÍSTICA}

Aquí se presentan los resultados técnicos obtenidos en el estudio, a fin de dar una más completa visión de los hallazgos:

\subsection{Muestra de Normalización}

Se buscó que las pruebas fueran normalizadas en todos los niveles de edad y para todos los grados que cubre el test a partir del 3er.Grado de Primaria, sobre muestra en número suficiente y, en lo posible representativas de la población de Lima Metropolitana, tomando como referencias los indicadores de número de alumnos matriculados en Centros Educativos de Lima Metropolitana (Oficina de Estadística del Ministerio de Educación, Lima, 1994). Las variables que sirvieron para conformar la muestra fueron: EDAD, SEXO, GRADO ESCOLAR, NIVEL SOCIOECONOMICO.

- EDAD: Se consideró el año completo de una edad a partir de la fecha de Nacimiento del alumno y comprendió desde por ejemplo 9.0 a 9.11 para incluirlo como 9 años. 


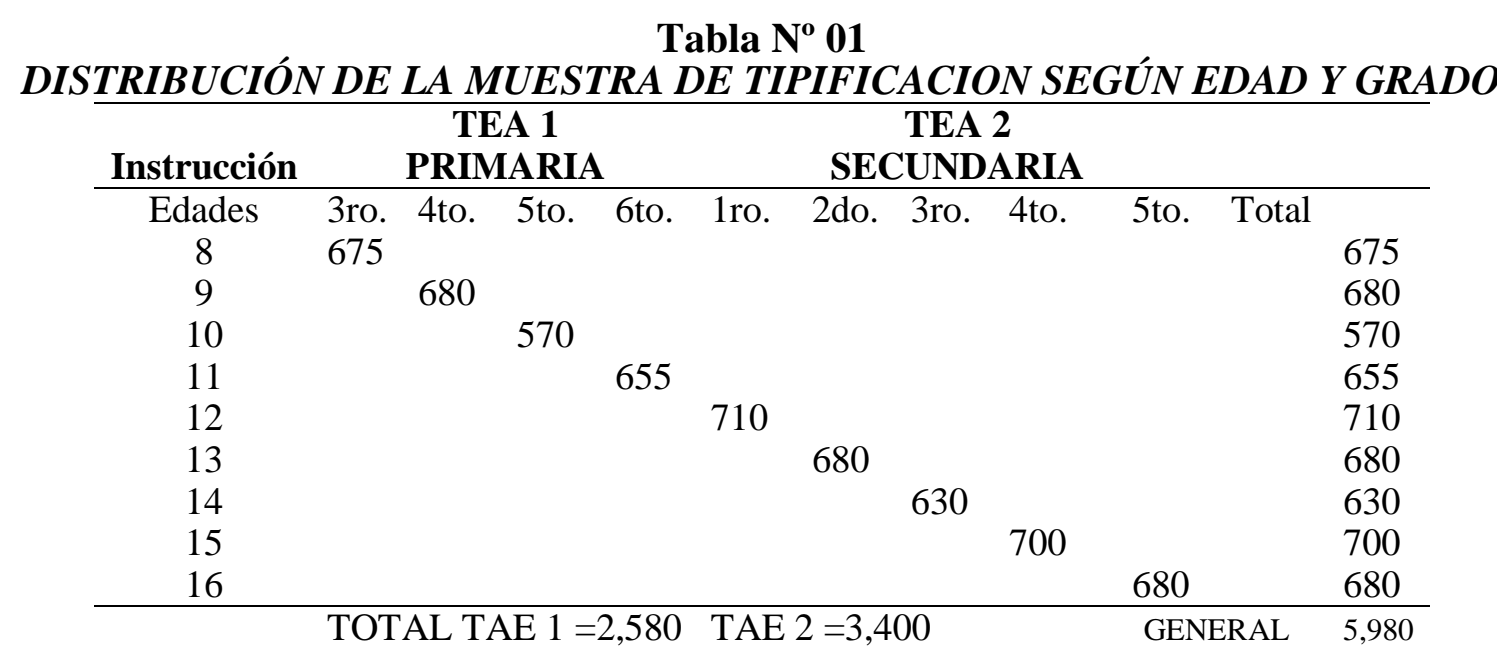

- SEXO: Estuvieron representados en forma homogénea escolares de ambos sexos.

- GRADO ESCOLAR: Se seleccionaron los alumnos considerando el grado escolar al que pertenecían en la fecha del examen, todos a la mitad del grado escolar por ser fecha de recolección de datos.

- NIVEL SOCIOECONOMICO: Se incluyó en la muestra centros educativos de todos los estratos socioeconómicos tanto estatales como particulares, considerando que dicha inclusión comprenderían diferentes grados en los niveles evaluados.

Él tipo de muestreo, estratificado y proporcional, permitió obtener la Muestra General de Tipificación, que según edad y grado escolar se presenta a continuación en la Tabla No. 01 Según estos, datos las, puntuaciones directas alcanzadas por los alumnos, sirvieron. para obtener una escala común de C.I. consecuentemente el lector usará las Edades para obtener los índices del C.I. y para los grados escolares trabajará mejor con Percentiles.

\subsection{Confiabilidad}

Uno de los estudios más impollantes sobre una prueba psicológica se refiere a la Confiabilidad. Ella alude a la Consistencia de las Puntuaciones; es decir, estadísticamente debe indicar la estabilidad o precisión de la medida y el grado en el que están libres de errores causal es o aleatorios.

Durante el trabajo de estandarización se llevaron a cabo estudios de Fiabilidad con diversos grupos y para los dos niveles del Test, recogiendo muestras de diferentes grados escolares. Se calculó el Coeficiente de Confiabilidad a partir de las puntuaciones directas de la prueba, usando el método Par - impar (split - half) y la corrección para su longitud total se hizo usando la fórmula de Spearman - Brown. 
TABLA N ${ }^{\circ} 02$

DESCRIPCIÓN DE LA MUESTRA DE TIPIFICACION POR NIVELES Y GRADO EN CADA UNO DE LOS FACTORES EVALUADOS CON MEDIAS Y DESVIACIÓN ESTANDAR

\begin{tabular}{|c|c|c|c|c|c|c|c|c|}
\hline \multicolumn{9}{|c|}{ TAE NIVEL 1} \\
\hline & $\begin{array}{c}\text { VEF } \\
X\end{array}$ & $\begin{array}{l}\text { BAL } \\
\text { S. }\end{array}$ & $\begin{array}{r}\text { RAZOI } \\
X\end{array}$ & $\begin{array}{l}\text { MIEN } \\
\text { S. }\end{array}$ & $\begin{array}{c}\text { CAL } \\
\text { X }\end{array}$ & $\begin{array}{l}\text { JLO } \\
\text { S. }\end{array}$ & & $\begin{array}{l}\mathrm{AL} \\
. \mathrm{S} .\end{array}$ \\
\hline 8 años & 22.80 & 6.10 & 15.80 & 4.24 & 15.50 & 6.80 & 54.16 & 13.50 \\
\hline 9 años & 25.69 & 7.05 & 17.09 & 4.14 & 19.90 & 6.19 & 62.30 & 14.10 \\
\hline 10 años & 28.92 & 8.09 & 18.03 & 4.07 & 21.64 & 6.95 & 66.70 & 14.50 \\
\hline 11 años & 30.05 & 8.15 & 19.21 & 3.80 & 23.80 & 7.03 & 73.40 & 13.80 \\
\hline
\end{tabular}

TAE NIVEL 2

\begin{tabular}{ccccccccc}
\hline & \multicolumn{2}{c}{ VERBAL } & \multicolumn{2}{c}{ RAZONAMIEN } & \multicolumn{2}{c}{ CALCULO } & \multicolumn{2}{c}{ TOTAL } \\
& \multicolumn{2}{c}{ X D.S. } & \multicolumn{2}{c}{ X D.S. } & \multicolumn{2}{c}{ X D.S. } & \multicolumn{2}{c}{ X D.S. } \\
\hline 12 años & 21.50 & 6.16 & 12.04 & 6.30 & 12.39 & 5.60 & 46.12 & 12.80 \\
13 años & 23.80 & 5.70 & 15.27 & 6.15 & 14.80 & 5.90 & 54.10 & 13.04 \\
14 años & 26.40 & 6.34 & 17.34 & 6.04 & 16.88 & 4.88 & 63.50 & 13.14 \\
15 años & 28.30 & 6.02 & 18.61 & 5.18 & 18.10 & 4.58 & 67.02 & 13.38 \\
16 años & 30.96 & 5.90 & 21.35 & 5.60 & 21.40 & 5.10 & 74.02 & 13.1 \\
\hline
\end{tabular}

TABLA N ${ }^{\circ} 03$

COEFICIENTES DE CONFIABILIDAD PARA EL T.A.E 1

\begin{tabular}{|c|c|c|c|c|}
\hline & 3er. Grado & 4to. Grado & 5to. Grado & 6to. Grado \\
\hline VERBAL & 0.80 & 0.87 & 0.89 & 0.88 \\
\hline RAZONAMIENTO & 0.83 & 0.80 & 0.82 & 0.84 \\
\hline CALCULO & 0.91 & 0.94 & 0.95 & 0.95 \\
\hline TOTAL & 0.93 & 0.92 & 0.93 & 0.90 \\
\hline
\end{tabular}

TABLA No 04

ERRORES TIPICOS DE MEDIDA DEL T.A.E. NIVEL 1

\begin{tabular}{|l|c|c|c|c|}
\hline & 3er. Grado & 4to. Grado & 5to. Grado & 6to. Grado \\
\hline VERBAL & 2.72 & 2.54 & 2.68 & 2.82 \\
\hline RAZONAMIENTO & 1.74 & 1.85 & 1.72 & 1.52 \\
\hline CALCULO & 2.04 & 1.51 & 1.55 & 1.57 \\
\hline TOTAL & 3.57 & 3.98 & 3.83 & 4.36 \\
\hline $\begin{array}{l}\text { EEM TOTAL } \\
\text { (al 68\% Confianza) }\end{array}$ & 4 & 4 & 4 & 4 \\
\hline
\end{tabular}

TABLA No 05

COEFICIENTES DE CONFIABILIDAD PARA EL T.A.E. 2

\begin{tabular}{lcccc}
\hline & VERBAL & RAZONAMIENTO & CALCULO & TOTAL \\
\hline $1^{\circ}$ Secund. & 0.82 & 0.91 & 0.89 & 0.90 \\
$2^{\circ}$ Secund. & 0.84 & 0.90 & 0.88 & 0.91 \\
$3^{\circ}$ Secund. & 0.86 & 0.89 & 0.86 & 0.90 \\
$4^{\text {o Secund. }}$ & 0.87 & 0.88 & 0.84 & 0.91 \\
$5^{\circ}$ Secund. & 0.89 & 0.91 & 0.89 & 0.92 \\
\hline
\end{tabular}


TABLA N ${ }^{\circ} 06$

ERRORES TIPICOS DE MEDIDA PARA EL T.A.E. 2

\begin{tabular}{lcccc}
\hline & VERBAL & RAZONAMIENTO & CALCULO & TOTAL \\
\hline $1^{\circ}$ Secund. & 2.61 & 1.89 & 1.85 & 4.04 \\
$2^{\circ}$ Secund. & 2.28 & 1.94 & 2.04 & 3.91 \\
$3^{\circ}$ Secund. & 2.10 & 2.00 & 1.82 & 4.15 \\
$4^{\circ}$ Secund. & 2.17 & 1.79 & 1.83 & 4.01 \\
$5^{\circ}$ Secund. & 1.95 & 1.69 & 1.70 & 3.70 \\
\hline
\end{tabular}

EI EEM TOTAL a nivel del $68 \%$ de confianza será de 4 puntos

\subsection{Validez}

La validez es, a decir de Tyler (1982), la consideración mas importante que se debe tener en la construcción y empleo de una prueba psicológica. Validez hace referencia al grado en que una prueba mide lo que intenta medir.

Además del procedimiento de análisis factorial, técnica que fue usada en la versión original del test de Thurstone, que constituye antecedente del TAE, existen otros procedimientos para estudiar la validez de un test psicológico. Aquí se utilizo la medida obtenida en una situación real de aprendizaje escolar vía el rendimiento académico expresado en NOTAS ESCOLARES como una medida de criterio. La correlación entre los puntajes de la prueba y dicha medida de criterio, permitió obtener los siguientes Coeficientes de Validez:

\section{TABLA N ${ }^{\circ} 08$ \\ COEFICIENTES DE CORRELACION COMO INDICE DE VALIDEZ \\ T.E.A. NIVEL 2}

$\begin{array}{clcccc}\text { Muestra } & \begin{array}{c}\text { Notas de } \\ \text { cursos }\end{array} & \text { VERBAL } & \text { RAZONAMIENTO } & \text { CALCULO } & \text { TOTAL } \\ \text { n }=169 & \text { Lenguaje } & 0.42 & 0.40 & 0.27 & 0.49 \\ \text { 1ero. } & \text { Matemáticas } & 0.34 & 0.39 & 0.40 & 0.44 \\ \text { Secund. } & \text { X General } & 0.42 & 0.40 & 0.38 & 0.51 \\ \mathrm{n}=180 & \text { Lenguaje } & 0.40 & 0.44 & 0.30 & 0.39 \\ \text { 3ero. } & \text { Matemáticas } & 0.33 & 0.40 & 0.41 & 0.45 \\ \text { Secund. } & \text { X General } & 0.44 & 0.42 & 0.40 & 0.55 \\ \mathrm{n}=166 \mathrm{X} & \text { Final } & 0.40 & 0.41 & 0.43 & 0.52\end{array}$


TABLA N 09

CORRELACIONES ENTRE LOS FACTORES T.E.A. Y FACTOR G

\begin{tabular}{llccccc}
\hline NIVEL & TEST & Grado & VERBAL & RAZONAM & CALCULO & TOTAL \\
\hline TAE 1 & $\begin{array}{c}\text { Factor } \\
\text { "G" 1 }\end{array}$ & $\begin{array}{c}\text { 3ro. } \\
\text { Prim. }\end{array}$ & 0.34 & 0.58 & 0.55 & 0.54 \\
\hline TAE 2 & $\begin{array}{l}\text { Factor } \\
\text { "G" 2 }\end{array}$ & $\begin{array}{c}\text { 1ro. } \\
\text { Secund. }\end{array}$ & 0.30 & 0.55 & 0.49 & 0.57 \\
\hline & $\begin{array}{c}\text { 2do. } \\
\text { Secund. }\end{array}$ & 0.31 & 0.54 & 0.53 & 0.55 \\
\hline
\end{tabular}

Asimismo, se llevaron a cabo otros tipos de análisis pero usando como criterios pruebas validadas tales como el test Factor G Serie abreviada que fue usada con el grupo de 8 años, $(n=120)$ y el Test Factor $\mathrm{G}$ - Forma 2, adaptada por la Dra. Nelly Ugarriza para el Instituto de Investigaciones Psicológicas de la Universidad Nacional Mayor de San Marcos, que lo utilizamos con escolares de [2 $(n=155)$ y 13 años $(n=130)$ y cuyos resultados se presentan a continuación:

\section{NORMAS PARA LA APLICACIÓN DE LAS PRUEBAS}

\subsection{Instrucciones Generales}

Seguir todas las indicaciones generales útiles a todo test psicológico tales como:

a) Estar familiarizado con las instrucciones de la prueba y el manejo del material de tests (cuadernillo, Hoja de respuestas ).

b) Estar al tanto de los tipos de items que tiene cada nivel del TAE y la forma cómo deben consignarse las respuestas en la Hoja preparada para este fin.

c) Poner mucho cuidado en la lectura de instrucciones usando los datos que aparecen en la misma prueba de modo que el examinado alcance un claro entendimiento de la tarea a realizar.

d) Los ejemplos que aparecen en los cuadernillos deben resolverse, ya que son valiosos datos de información sobre la forma en que se va a proceder durante el desarrollo de la prueba que se evalúa.

e) Cuide que el ambiente de examen cuente con los requisitos que se exigen a todo examen con tests. Use un tono de voz uniforme señalando claramente el momento de INICIO y FIN de cada prueba.

\subsection{Material de Examen}

1. Manual adaptado con las instrucciones de examen

2. Cuadernillos

3. Lápiz y borrador

4. Hojas de Respuesta (Una para cada Nivel)

5. Cronómetro para adecuado control del tiempo.

6. Plantilla de corrección

\subsection{Procedimiento Durante el Examen}

Empiece entregando lápiz y la Hoja de respuestas y pida a cada alumno que anoten los datos que se le solicitan; luego. señale la adecuada forma de responder: 


\section{CORRECTO ERROR}

Deben sombrear totalmente y dentro de las líneas, para que al colocar la plantilla sobre la clave observé a través de la perforación la marca hecha por el examinado facilitando de este modo el proceso de calificación.

Después entregue a cada evaluado el cuadernillo y pida que lo ubique en el lado izquierdo de la carpeta de modo que les facilite el anotar las respuestas. Posteriormente el examinador leerá las instrucciones en voz alta mientras que los evaluados lo hacen con la vista siguiendo la lectura desde sus cuadernillos.

Todas las dudas deben aclararse antes de iniciar cada prueba, pero de ningún modo. se darán nuevos ejemplos o aclaraciones ajenas a las que aparecen en el cuadernillo.

\section{TIEMPOS DE APLICACIÓN DE LAS PRUEBAS}

\begin{tabular}{lll} 
TAE 1: & Subtest & Tiempo sugerido \\
& Dibujos & 4 minutos \\
& Palabras diferentes & 4 minutos \\
& Vocabulario & 5 minutos \\
& Razonamiento & 8 minutos \\
& Cálculo & 5 minutos \\
\hline & TOTAL & 22 minutos \\
TAE 2: & Subtest & Tiempo requerido \\
& Palabras diferentes & 6 minutos \\
& Vocabulario & 8 minutos \\
& Series de Números & 10 minutos \\
& Series de letras & 6 minutos \\
& Cálculo & 12 minutos \\
\hline & TOTAL & 42 minutos
\end{tabular}

\subsection{Instrucciones Especificas para T.A.E. 1}

\section{Subtest DIBUJOS}

Observa figuras del ejemplo 1. Debes encontrar la figura del PERRO (Pausa) Bien observen que arriba tiene una letra B por lo tanto, la letra B es la respuesta correcta del ejemplo. En la hoja de respuestas marca la letra ... B"

"Observa el ejemplo 2 y busca la HOJA" "Dime que letra es la correcta"(Pausa).

"Ahora lo marcas en la hoja de respuestas" (supervisar).

"Ahora vamos a trabajar en la siguiente fila el ejercicio 3. Busca la MARIPOSA.

Cuando la encuentres mira la letra que tiene encima y, en la hoja de respuestas harás la anotación respectiva" (supervise). Luego continúe con las indicaciones que aparecen en el cuadernillo de instrucciones. 
Palabras requeridas para evaluar dibujos

$\begin{array}{ll}\text { 4. INSECTO } & \text { 5. BEBE } \\ \text { 6. REFRESCO } & \text { 7. EQUIPAJE } \\ \text { 8. BALLESTA } & \text { 9. CEREAL } \\ \text { 10. LABRIEGO } & \text { 11. RED } \\ \text { 12. GAVILAN } & \text { 13. PAQUIDERMO } \\ \text { 14. PERCANCE } & \text { 15. ARTESANO } \\ \text { 16. LA MALLA } & \text { 17. GALENO } \\ \text { 18. ALERO } & \end{array}$

\section{Subtest PALABRAS DIFERENTES}

Pida que volteen la hoja y busquen en el cuadernillo donde dice PALABRAS DIFERENTES. Lea Ud. en voz alta las instrucciones y los evaluados deben seguir con la vista dicha lectura. Proceda a que desarrollen los ejemplos, supervise el trabajo y dé la orden de inicio y término de esta parte de la prueba.

\section{Subtest VOCABULARIO}

Diga "En cada ejercicio tienes que buscar la palabra que significa lo mismo que la primera que está en mayúsculas" Siga exactamente las indicaciones que aparecen en el cuadernillo y recuerde la forma de responder en la Hoja de Respuestas.

\section{Subtest RAZONAMIENTO}

Igualmente pida que ubiquen esta parte de la prueba y lea claramente las instrucciones. "En cada ejercicio tienes que buscar el dibujo que es diferente".

\section{Subtest CALCULO}

Lea pausadamente las instrucciones impresas en el cuadernillo. Diga: "Aquí hay algunas sumas que ha hecho un alumno llamado Juan Pero Juan no ha hecho Todas las sumas bien y algunas están mal. Tú debes sumar de memoria y colocar B (Bien) ó M (Mal) en la hoja de respuestas, según el resultado. No escribas nada en el cuadernillo"

No olvide leer todas las instrucciones según aparecen en el cuadernillo antes de dar la orden de empezar.

Al final del tiempo exacto de 5 minutos diga "ALTO" "Fin de la prueba" y recoja Hojas de Respuestas, cuadernillos y lápices.

\subsection{Instrucciones Especificas para el T.A.E. 2}

Deben leerse exactamente cada una de las instrucciones que aparecen en el cuadernillo del test usando un tono de voz clara y cuidando mantener interesado al sujeto durante el desarrollo de la prueba.

Subtest PALABRAS DIFERENTES "Observa los ejercicios que hay aquí más abajo.

Todos ellos se han hecho con palabras. En cada ejercicio hay 4 palabras que se parecen en algo y otra que es diferente. La tarea consiste en buscar la palabra diferente".

Se sigue con ejemplos. Se recuerda la forma de contestar en la Hoja de Respuestas. Se aclaran dudas y se empieza la prueba cuidando el control del tiempo de 6 minutos.

\section{Subtest VOCABULARIO}

"En cada ejercicio tienes que buscar la palabra que significa lo mismo que la primera. Tiempo: 8 minutos.

\section{Subtest SERIES DE NUMEROS}

Lea exactamente las instrucciones del cuadernillo Diga "En cada ejercicio debes observar el orden que tienen los números de la izquierda para ver cuál es el número que seguiría ese 
orden, si continuase, y buscarlo entre los números de la derecha" Trabaje los ejemplos con el examinado. Tenga seguridad que se entendió la tarea a realizar. Dé la orden de inicio. Controle exactamente los 10 minutos de trabajo y luego diga "ALTO".

\section{Subtest SERIES DE LETRAS}

Diga: "En cada ejercicio debes observar el orden que tienen las letras, para ver cuál es la que seguiría ese orden si continuase; luego señala en la Hoja de Respuestas el espacio debajo de esa letra. En esta prueba las letras son minúsculas y no se emplean las letras compuestas ( ch,1l,rr)

Desarrolle ejemplos. Aclare dudas. Otorgue los 6 minutos para trabajar esta parte del Test.

\section{Subtest CALCULO}

Lea pausadamente las instrucciones del cuadernillo. Desarrolle el 1er. Ejemplo con el sujeto. Pida que resuelva los ejemplos 2, 3 y 4 leyendo las instrucciones existentes para su aplicación. Aclare dudas. Dé la orden de inicio. Controle exactamente los 12 minutos de prueba. Diga "ALTO" Retire todo el material por que finalizó el examen.

\section{CALIFICACIÓN DE LAS PRUEBAS T.A.E. NIVELES 1 Y 2}

Antes de colocar la plantilla sobre la Hoja de Respuestas revise para encontrar preguntas con dos respuestas. De encontrar alguna se anulará dicha pregunta.

Coloque la plantilla perforada sobre la Hoja de Respuestas cuidando de que la posición sea la correcta. Luego sume para cada prueba las respuestas que coinciden con la perforación y otorgue para cada una UN PUNTO haciendo un total por prueba. La puntuación directa en todas las pruebas (excepto en Cálculo del TAE-I) es igual al número de aciertos.

En Cálculo se tendrán en cuenta los errores, por lo que el puntaje directo resulta de los aciertos menos los errores, Los puntajes se convertirán en puntuaciones normalizadas de c.1. o Percentiles según sea el caso específico para cada Nivel del Test de Aptitudes Escolares 1 ó 2.

\section{NORMALIZACION}

A continuación se ofrecen las Normas que se han elaborado para Lima Metropolitana tanto del TAE I como del TAE 2. Se han diseñado Normas de C.I. de desviación con MEDIA 100 Y DESVIACIÓN STANDARD 16.

Las tablas que corresponden al TAE 1 son: la 1 (aptitud verbal); la 2, (Aptitud de Razonamiento); la 3 (aptitud de cálculo) y la tabla 4 permite obtener la aptitud general para usarse con escolares entre 8 y 11 años.

Las tablas que corresponden al TAE 2 son: la 5 (aptitud verbal) la 6 (Razonamiento); la 7 (cálculo) y la tabla 8 mide la aptitud general de los escolares entre 12 y 16 años.

Asimismo se han diseñado tablas basadas en Normas Percentiles las que permiten comparaciones entre los escolares de acuerdo a su grado escolar. Así, las tablas de la 9 a la 12 pertenecen al TAE 1 en todos los niveles de aptitud evaluados; y, las tablas de 13 a 16 corresponden al TAE 2.

\section{INTERPETACION DE RESULTADOS}

En la interpretación de los c.1. que brinda el TAE se debe considerar que lo que se ha medido se refiere al nivel de aptitud presente que se requiere para desempeñar tareas escolares según una edad determinada.

Los C.I. que se obtengan pueden ser interpretados según la calificación siguiente: 
COEFICIENTES DE APTITUD

\begin{tabular}{ccc}
\hline C.I. & Categoría & Diagnostica \\
\hline $129 \mathrm{a}+$ & Aptitud & MUY SUPERIOR \\
$119-128$ & Aptitud & SUPERIOR \\
$109-118$ & Aptitud & ARRIBA DEL PROMEDIO \\
$89-108$ & Aptitud & NORMAL PROMEDIO \\
$79-88$ & Aptitud & DEBAJO DEL PROMEDIO \\
$69-78$ & Aptitud & DEFINITIVAMENTE BAJA \\
$49-68$ & Aptitud & DEFICIENTE \\
\hline
\end{tabular}

Si el patrón de análisis fuese escogiendo Puntuaciones Percentiles se usara el siguiente cuadro

\begin{tabular}{ccc}
\hline & $96-99$ & Dotación muy alta \\
& $75-95$ & Aptitud Superior \\
Percentil & $26-74$ & Aptitud Normal \\
& $25-06$ & Aptitud Baja \\
& 05 ó - & Aptitud Deficiente \\
\hline
\end{tabular}

BAREMOS DEL TEST DE APTITUDES ESCOLARES

TABLAS DEL T.A.E. 1

C.I. FACTOR VERBAL PARA 8,9,10 Y 11 AÑOS

C.I. FACTOR RAZONAMIENTO

C.I. FACTOR CALCULO

C.I. FACTORS GENERAL DE APTITUD ESCOLAR

TABLAS PERCENTILES POR NIVEL DE EDAD

TABLAS DEL T.A.E. 2

C.I. FACTOR VERBAL PARA 12, 13,14, 15 Y 16 AÑOS

C.I. FACTOR RAZONAMIENTO

C.I. FACTOR CALCULO

C.I. FACTORS GENERAL DE APTITUD ESCOLAR

TABLAS PERCENTILES POR NIVEL DE EDAD 
TABLA N $\mathbf{N}^{\circ} 1$

TAE 1 FACTOR VERBAL CONVERSIÓN DEL PUNTAJE DIRECTO EN C.I. SEGÚN EDADES

\begin{tabular}{|c|c|c|c|c|c|c|c|c|c|c|c|}
\hline \multicolumn{6}{|c|}{ EDADES } & \multicolumn{6}{|c|}{ EDADES } \\
\hline P.D. & 8 & 9 & 10 & 11 & P.D. & P.D. & 8 & 9 & 10 & 11 & P.D. \\
\hline 50 & - & - & 144 & 140 & 50 & 27 & 120 & 409 & 98 & 94 & 27 \\
\hline 49 & - & - & 142 & 138 & 49 & 26 & 117 & 103 & 96 & 92 & 226 \\
\hline 48 & - & - & 140 & 136 & 48 & 25 & 115 & 100 & 94 & 90 & 25 \\
\hline 47 & & 150 & 138 & 134 & 47 & 24 & 110 & 98 & 92 & 87 & 24 \\
\hline 46 & - & 148 & 136 & 132 & 46 & 23 & 108 & 96 & 90 & 85 & 23 \\
\hline 45 & - & 147 & 134 & 130 & 45 & 22 & 102 & 94 & 88 & 83 & 22 \\
\hline 44 & - & 145 & 131 & 128 & 44 & 21 & 100 & 92 & 86 & 81 & 21 \\
\hline 43 & - & 143 & 129 & 126 & 43 & 20 & 98 & 90 & 84 & 79 & 20 \\
\hline 42 & - & 140 & 127 & 124 & 42 & 19 & 96 & 88 & 81 & 77 & 19 \\
\hline 41 & - & 138 & 125 & 122 & 41 & 18 & 94 & 86 & 79 & 74 & 18 \\
\hline 40 & - & 136 & 123 & 120 & 40 & 17 & 92 & 84 & 76 & 72 & 17 \\
\hline 39 & 150 & 132 & 121 & 117 & 39 & 16 & 91 & 82 & 74 & 70 & 16 \\
\hline 38 & 148 & 130 & 120 & 114 & 38 & 15 & 88 & 80 & 71 & 68 & 15 \\
\hline 37 & 145 & 128 & 118 & 112 & 37 & 14 & 85 & 78 & 69 & 66 & 14 \\
\hline 36 & 142 & 126 & 116 & 110 & 36 & 13 & 83 & 77 & 67 & 64 & 13 \\
\hline 35 & 140 & 124 & 114 & 108 & 35 & 12 & 80 & 75 & 65 & 62 & 12 \\
\hline 34 & 137 & 123 & 112 & 106 & 34 & 11 & 78 & 73 & 63 & 60 & 11 \\
\hline 33 & 135 & 121 & 110 & 104 & 33 & 10 & 75 & 71 & 60 & 58 & 10 \\
\hline 32 & 133 & 120 & 108 & 102 & 32 & 9 & 73 & 68 & 58 & 56 & 9 \\
\hline 31 & 130 & 118 & 106 & 100 & 31 & 8 & 70 & 66 & 56 & 54 & 8 \\
\hline 30 & 128 & 116 & 104 & 99 & 30 & 7 & 69 & 65 & 55 & 52 & 7 \\
\hline 29 & 124 & 114 & 102 & 98 & 29 & 6 & 68 & 64 & 54 & 50 & 6 \\
\hline 28 & 122 & 112 & 100 & 96 & 28 & 5 & 67 & 63 & 53 & 48 & 5 \\
\hline
\end{tabular}


TABLA N $\mathbf{N}^{\circ} 2$

TAE 1 FACTOR RAZONAMIENTO CONVERSIÓN DEL PUNTAJE DIRECTO EN C.I. SEGÚN EDADES

\begin{tabular}{|c|c|c|c|c|c|c|c|c|c|c|c|}
\hline \multicolumn{2}{|c|}{ EDADES } & \multicolumn{1}{c|}{ EDADES } \\
\hline P.D. & 8 & 9 & 10 & 11 & P.D. & P.D. & 8 & 9 & 10 & 11 & P.D. \\
\hline 30 & 152 & 147 & 145 & 144 & 30 & 15 & 101 & 94 & 90 & 87 & 15 \\
29 & 149 & 145 & 143 & 140 & 29 & 14 & 98 & 90 & 86 & 80 & 14 \\
28 & 146 & 141 & 139 & 136 & 28 & 13 & 96 & 87 & 82 & 78 & 13 \\
27 & 143 & 137 & 135 & 132 & 27 & 12 & 94 & 84 & 79 & 74 & 12 \\
26 & 140 & 133 & 131 & 128 & 26 & 11 & 92 & 81 & 76 & 70 & 11 \\
25 & 137 & 129 & 127 & 123 & 25 & 10 & 90 & 77 & 72 & 66 & 10 \\
24 & 134 & 125 & 122 & 119 & 24 & 9 & 87 & 74 & 68 & 62 & 9 \\
23 & 131 & 120 & 117 & 115 & 23 & 8 & 84 & 71 & 64 & 58 & 8 \\
22 & 128 & 118 & 113 & 112 & 22 & 7 & 81 & 68 & 60 & 54 & 7 \\
21 & 124 & 114 & 110 & 109 & 21 & 6 & 76 & 64 & 56 & 50 & 6 \\
20 & 120 & 110 & 108 & 105 & 20 & 5 & 71 & 61 & 52 & 46 & 5 \\
19 & 116 & 107 & 104 & 100 & 19 & 4 & 67 & 57 & 48 & 42 & 4 \\
18 & 112 & 103 & 100 & 96 & 18 & 3 & 63 & 53 & 44 & 38 & 3 \\
16 & 109 & 100 & 97 & 92 & 17 & 2 & 59 & 50 & 40 & 34 & 2 \\
\hline
\end{tabular}


TABLA N $\mathbf{N}^{\circ} 3$

TAE 1 FACTOR CALCULO CONVERSIÓN DEL PUNTAJE DIRECTO EN C.I. SEGÚN EDADES

\begin{tabular}{|c|c|c|c|c|c|c|c|c|c|c|c|}
\hline \multicolumn{6}{|c|}{ EDADES } & \multicolumn{6}{|c|}{ EDADES } \\
\hline P.D. & 8 & 9 & 10 & 11 & P.D. & P.D. & 8 & 9 & 10 & 11 & P.D. \\
\hline 43 & - & - & - & - & 43 & 25 & 134 & 118 & 109 & 106 & 25 \\
\hline 42 & - & - & - & - & 42 & 24 & 131 & 116 & 106 & 102 & 24 \\
\hline 41 & - & - & - & 150 & 41 & 23 & 128 & 112 & 103 & 100 & 23 \\
\hline 40 & - & - & 150 & 148 & 40 & 22 & 125 & 109 & 100 & 98 & 22 \\
\hline 39 & - & - & 148 & 145 & 39 & 21 & 123 & 106 & 99 & 96 & 21 \\
\hline 38 & - & - & 145 & 143 & 38 & 20 & 120 & 102 & 97 & 92 & 20 \\
\hline 37 & - & - & 143 & 141 & 37 & 19 & 116 & 100 & 95 & 90 & 19 \\
\hline 36 & - & - & 140 & 138 & 36 & 18 & 112 & 97 & 93 & 87 & 18 \\
\hline 35 & - & 150 & 138 & 135 & 35 & 17 & 108 & 94 & 90 & 85 & 17 \\
\hline 34 & - & 146 & 136 & 132 & 34 & 16 & 104 & 90 & 88 & 82 & 16 \\
\hline 33 & - & 142 & 133 & 128 & 33 & 15 & 100 & 87 & 86 & 80 & 15 \\
\hline 32 & - & 138 & 130 & 125 & 32 & 14 & 99 & 84 & 84 & 77 & 14 \\
\hline 31 & - & 135 & 126 & 123 & 31 & 13 & 97 & 80 & 82 & 74 & 13 \\
\hline 30 & - & 132 & 123 & 120 & 30 & 12 & 95 & 78 & 80 & 71 & 12 \\
\hline 29 & 150 & 129 & 120 & 117 & 29 & 11 & 93 & 75 & 78 & 68 & 11 \\
\hline 28 & 146 & 126 & 117 & 113 & 28 & 10 & 91 & 72 & 75 & 65 & 10 \\
\hline 27 & 142 & 122 & 115 & 110 & 27 & 9 & 89 & 69 & 72 & 62 & 9 \\
\hline 26 & 138 & 120 & 111 & 108 & 26 & 8 & 87 & 66 & 70 & 60 & 8 \\
\hline
\end{tabular}


TABLA N $\mathbf{N}^{\circ}$

TAE 1 PUNTAJE TOTAL CONVERSIÓN DEL PUNTAJE DIRECTO EN C.I. SEGÚN EDADES

\begin{tabular}{|c|c|c|c|c|c|c|c|c|c|c|c|}
\hline \multicolumn{6}{|c|}{ EDADES } & \multicolumn{6}{|c|}{ EDADES } \\
\hline P.D. & 8 & 9 & 10 & 11 & P.D. & P.D. & 8 & 9 & 10 & 11 & P.D. \\
\hline 120 & - & - & - & - & 120 & 63 & 113 & 100 & 96 & 90 & 63 \\
\hline 117 & - & - & - & - & 117 & 60 & 109 & 99 & 93 & 86 & 60 \\
\hline 114 & - & - & 152 & 146 & 114 & 57 & 105 & 97 & 91 & 82 & 57 \\
\hline 111 & - & - & 148 & 144 & 111 & 54 & 100 & 95 & 89 & 78 & 54 \\
\hline 108 & - & 152 & 144 & 142 & 108 & 51 & 99 & 91 & 86 & 74 & 51 \\
\hline 105 & - & 148 & 141 & 138 & 105 & 48 & 97 & 88 & 83 & 70 & 48 \\
\hline 102 & - & 145 & 138 & 134 & 102 & 45 & 95 & 85 & 80 & 66 & 45 \\
\hline 99 & & 142 & 135 & 130 & 99 & 42 & 93 & 81 & 76 & 62 & 42 \\
\hline 96 & 142 & 138 & 132 & 128 & 96 & 39 & 90 & 78 & 72 & 58 & 39 \\
\hline 93 & 140 & 135 & 129 & 124 & 93 & 36 & 86 & 75 & 69 & 51 & 36 \\
\hline 90 & 138 & 132 & 126 & 120 & 90 & 33 & 83 & 71 & 66 & 51 & 33 \\
\hline 87 & 136 & 129 & 123 & 117 & 87 & 30 & 79 & 67 & 62 & 47 & 30 \\
\hline 84 & 132 & 126 & 120 & 115 & 84 & 27 & 76 & 64 & 59 & 41 & 27 \\
\hline 81 & 130 & 123 & 117 & 111 & 81 & 24 & 73 & 60 & 56 & 39 & 24 \\
\hline 78 & 128 & 120 & 113 & 108 & 78 & 21 & 70 & 56 & 53 & & 21 \\
\hline 75 & 126 & 116 & 111 & 104 & 75 & 18 & 67 & 51 & 50 & & 18 \\
\hline 72 & 123 & 112 & 108 & 100 & 72 & 15 & 62 & 48 & & & 15 \\
\hline 69 & 120 & 107 & 104 & 97 & 69 & 12 & 59 & 46 & & & 12 \\
\hline 66 & 117 & 103 & 100 & 93 & 66 & 9 & 51 & 44 & & & 9 \\
\hline
\end{tabular}


TABLA N $\mathbf{N}^{\circ}$

TAE 2 FACTOR VERBAL CONVERSIÓN DEL PUNTAJE DIRECTO EN C.I. SEGÚN EDADES

\begin{tabular}{|c|c|c|c|c|c|c|c|c|c|c|c|}
\hline \multicolumn{6}{|c|}{ EDADES } & \multicolumn{6}{|c|}{ EDADES } \\
\hline P.D. & 12 & 13 & 14 & 15 & 16 & P.D. & 12 & 13 & 14 & 15 & 16 \\
\hline 50 & - & - & - & - & - & 27 & 115 & 108 & 98 & 98 & 93 \\
\hline 49 & - & - & - & - & - & 26 & 113 & 105 & 96 & 95 & 91 \\
\hline 48 & - & - & - & - & 156 & 25 & 107 & 102 & 94 & 93 & 89 \\
\hline 47 & - & - & - & - & 153 & 24 & 105 & 100 & 91 & 91 & 86 \\
\hline 46 & - & - & - & 153 & 150 & 23 & 102 & 98 & 89 & 89 & 82 \\
\hline 45 & - & - & 151 & 151 & 147 & 22 & 100 & 95 & 87 & 86 & 80 \\
\hline 44 & - & - & 148 & 148 & 144 & 21 & 99 & 93 & 84 & 83 & 77 \\
\hline 43 & - & 152 & 146 & 145 & 140 & 20 & 97 & 90 & 81 & 80 & 74 \\
\hline 42 & - & 147 & 144 & 142 & 136 & 19 & 95 & 88 & 78 & 77 & 71 \\
\hline 41 & - & 146 & 142 & 139 & 133 & 18 & 93 & 86 & 75 & 74 & 68 \\
\hline 40 & 150 & 144 & 140 & 136 & 130 & 17 & 91 & 83 & 72 & 71 & 65 \\
\hline 39 & 147 & 142 & 136 & 133 & 126 & 16 & 89 & 80 & 69 & 68 & 62 \\
\hline 38 & 145 & 138 & 132 & 130 & 122 & 15 & 86 & 78 & 65 & 65 & 59 \\
\hline 37 & 142 & 134 & 129 & 127 & 117 & 14 & 82 & 74 & 62 & 61 & 56 \\
\hline 36 & 139 & 132 & 128 & 124 & 115 & 13 & 79 & 71 & 59 & 57 & 53 \\
\hline 35 & 136 & 129 & 124 & 121 & 113 & 12 & 75 & 68 & 56 & 54 & 50 \\
\hline 34 & 133 & 127 & 121 & 117 & 110 & 11 & 73 & 65 & 53 & 51 & \\
\hline 33 & 131 & 125 & 118 & 115 & 107 & 10 & 70 & 62 & 50 & 47 & \\
\hline 32 & 129 & 122 & 115 & 113 & 105 & 9 & 67 & 58 & & & \\
\hline 31 & 126 & 120 & 111 & 111 & 102 & 8 & 64 & 54 & & & \\
\hline 30 & 123 & 118 & 108 & 108 & 100 & 7 & 61 & 51 & & & \\
\hline 29 & 120 & 115 & 104 & 104 & 97 & 6 & 58 & 48 & & & \\
\hline 28 & 118 & 112 & 100 & 100 & 95 & 5 & 55 & & & & \\
\hline
\end{tabular}


TABLA No 6

TAE 2 FACTOR RAZONAMIENTO CONVERSIÓN DEL PUNTAJE DIRECTO EN C.I. SEGÚN EDADES

\begin{tabular}{|c|c|c|c|c|c|c|c|c|c|c|c|}
\hline \multicolumn{6}{|c|}{ EDADES } & \multicolumn{6}{|c|}{ EDADES } \\
\hline P.D. & 12 & 13 & 14 & 15 & 16 & P.D. & 12 & 13 & 14 & 15 & 16 \\
\hline 30 & 153 & 152 & 152 & 139 & 128 & 16 & 113 & 104 & 98 & 93 & 87 \\
\hline 29 & 151 & 150 & 150 & 136 & 125 & 15 & 111 & 100 & 95 & 90 & 84 \\
\hline 28 & 148 & 146 & 146 & 133 & 122 & 14 & 107 & 98 & 92 & 87 & 80 \\
\hline 27 & 145 & 143 & 143 & 130 & 119 & 13 & 104 & 96 & 89 & 84 & 76 \\
\hline 26 & 142 & 140 & 140 & 127 & 116 & 12 & 100 & 93 & 86 & 81 & 73 \\
\hline 25 & 139 & 137 & 137 & 124 & 112 & 11 & 96 & 90 & 83 & 78 & 70 \\
\hline 24 & 136 & 133 & 133 & 121 & 109 & 10 & 93 & 87 & 80 & 75 & 67 \\
\hline 23 & 132 & 129 & 129 & 118 & 106 & 9 & 90 & 84 & 76 & 71 & 64 \\
\hline 22 & 129 & 126 & 126 & 115 & 103 & 8 & 86 & 81 & 73 & 68 & 61 \\
\hline 21 & 126 & 123 & 123 & 112 & 100 & 7 & 82 & 78 & 70 & 65 & 57 \\
\hline 20 & 123 & 120 & 120 & 109 & 98 & 6 & 78 & 75 & 67 & 62 & 54 \\
\hline 19 & 120 & 116 & 116 & 106 & 95 & 5 & 74 & 72 & 64 & 59 & 51 \\
\hline 18 & 117 & 112 & 112 & 103 & 92 & 4 & 70 & & & & \\
\hline 17 & 115 & 108 & 108 & 100 & 89 & & & & & & \\
\hline
\end{tabular}


TABLA No 7

TAE 2 FACTOR CALCULO CONVERSIÓN DEL PUNTAJE DIRECTO EN C.I. SEGÚN EDADES

\begin{tabular}{|c|c|c|c|c|c|c|c|c|c|c|c|}
\hline \multicolumn{6}{|c|}{ EDADES } & \multicolumn{6}{|c|}{ EDADES } \\
\hline P.D. & 12 & 13 & 14 & 15 & 16 & P.D. & 12 & 13 & 14 & 15 & 16 \\
\hline 30 & - & - & 139 & 136 & 130 & 15 & 111 & 102 & 95 & 92 & 85 \\
\hline 29 & - & - & 136 & 133 & 127 & 14 & 107 & 100 & 92 & 89 & 82 \\
\hline 28 & 152 & 150 & 133 & 130 & 123 & 13 & 102 & 98 & 89 & 86 & 79 \\
\hline 27 & 149 & 146 & 130 & 127 & 120 & 12 & 100 & 95 & 86 & 83 & 76 \\
\hline 26 & 146 & 142 & 127 & 124 & 117 & 11 & 98 & 92 & 83 & 80 & 73 \\
\hline 25 & 143 & 138 & 124 & 121 & 113 & 10 & 95 & 89 & 80 & 77 & 70 \\
\hline 24 & 140 & 134 & 121 & 118 & 110 & 9 & 92 & 86 & 77 & 74 & 67 \\
\hline 23 & 139 & 131 & 118 & 115 & 106 & 8 & 90 & 83 & 74 & 70 & 64 \\
\hline 22 & 134 & 127 & 115 & 112 & 103 & 7 & 87 & 80 & 71 & 67 & 61 \\
\hline 21 & 131 & 123 & 112 & 109 & 100 & 6 & 84 & 77 & 68 & 64 & 58 \\
\hline 20 & 128 & 119 & 109 & 106 & 98 & 5 & 81 & 74 & 65 & 61 & 55 \\
\hline 19 & 124 & 115 & 106 & 103 & 96 & 4 & 78 & 71 & 62 & 58 & 52 \\
\hline 18 & 121 & 11 & 103 & 100 & 94 & 3 & 75 & 68 & 59 & 55 & 49 \\
\hline 17 & 118 & 108 & 100 & 98 & 91 & 2 & 72 & 65 & 56 & 52 & 46 \\
\hline 16 & 115 & 105 & 98 & 95 & 88 & 1 & 69 & 62 & 53 & 49 & 43 \\
\hline
\end{tabular}


TABLA No 8

TAE 2 FACTOR CALCULO CONVERSIÓN DEL PUNTAJE DIRECTO EN C.I. SEGÚN EDADES

\begin{tabular}{|c|c|c|c|c|c|c|c|c|c|c|c|}
\hline \multicolumn{6}{|c|}{ EDADES } & \multicolumn{6}{|c|}{ EDADES } \\
\hline P.D. & 12 & 13 & 14 & 15 & 16 & P.D. & 12 & 13 & 14 & 15 & 16 \\
\hline 103 & - & - & - & 136 & 139 & 51 & 106 & 98 & 89 & 86 & 77 \\
\hline 100 & - & - & 146 & 133 & 135 & 48 & 103 & 95 & 86 & 83 & 74 \\
\hline 97 & - & - & 142 & 130 & 131 & 45 & 101 & 92 & 82 & 80 & 71 \\
\hline 94 & - & 151 & 138 & 127 & 127 & 42 & 97 & 89 & 78 & 77 & 68 \\
\hline 91 & 153 & 147 & 134 & 124 & 123 & 39 & 94 & 86 & 76 & 73 & 65 \\
\hline 88 & 149 & 143 & 130 & 121 & 119 & 36 & 90 & 83 & 73 & 69 & 62 \\
\hline 85 & 145 & 139 & 126 & 118 & 115 & 33 & 86 & 80 & 70 & 65 & 59 \\
\hline 82 & 141 & 135 & 122 & 115 & 111 & 30 & 82 & 77 & 67 & 61 & 56 \\
\hline 79 & 137 & 131 & 118 & 112 & 107 & 27 & 78 & 74 & 64 & 57 & 53 \\
\hline 76 & 133 & 127 & 114 & 109 & 103 & 24 & 74 & 70 & 60 & 53 & 49 \\
\hline 73 & 129 & 123 & 111 & 106 & 99 & 21 & 70 & 66 & 57 & 49 & 46 \\
\hline 70 & 125 & 119 & 107 & 103 & 96 & 18 & 66 & 62 & 54 & 45 & 43 \\
\hline 67 & 121 & 115 & 104 & 100 & 93 & 15 & 62 & 58 & 51 & 41 & 40 \\
\hline 64 & 118 & 111 & 101 & 98 & 90 & 12 & 57 & 54 & 48 & 37 & 37 \\
\hline 61 & 115 & 107 & 98 & 95 & 86 & 09 & 53 & 50 & 45 & 33 & 34 \\
\hline 58 & 112 & 103 & 95 & 92 & 83 & 06 & 49 & 46 & 42 & 29 & 31 \\
\hline 55 & 109 & 100 & 92 & 89 & 80 & & & & & & \\
\hline
\end{tabular}


TABLA No 9

TAE 1 FACTOR VERBAL PUNTUCIONES PERCNTILES POR GRADO ESCOLAR

\begin{tabular}{|c|c|c|c|c|c|c|c|c|c|c|c|}
\hline \multicolumn{6}{|c|}{ GRADOS ESCOLARES } & \multicolumn{6}{|c|}{ GRADOS ESCOLARES } \\
\hline P.D. & 3ro & 4 to & 5to & 6to & P.D. & P.D. & 3ro & 4 to & 5to & 6to & P.D. \\
\hline 49 & - & - & - & 99 & 49 & 28 & 92 & 77 & 50 & 40 & 28 \\
\hline 48 & - & - & - & 99 & 48 & 27 & 89 & 71 & 45 & 35 & 27 \\
\hline 47 & - & - & - & 98 & 47 & 26 & 85 & 57 & 40 & 31 & 26 \\
\hline 46 & - & - & - & 98 & 46 & 25 & 83 & 50 & 35 & 27 & 25 \\
\hline 45 & - & - & 99 & 97 & 45 & 24 & 73 & 45 & 31 & 21 & 24 \\
\hline 44 & - & - & 98 & 96 & 44 & 23 & 69 & 40 & 27 & 17 & 23 \\
\hline 43 & - & - & 96 & 95 & 43 & 22 & 55 & 35 & 23 & 15 & 22 \\
\hline 42 & - & - & 95 & 93 & 42 & 21 & 50 & 31 & 19 & 12 & 21 \\
\hline 41 & - & - & 94 & 92 & 41 & 20 & 45 & 27 & 16 & 10 & 20 \\
\hline 40 & - & - & 93 & 89 & 40 & 19 & 40 & 23 & 12 & 7 & 19 \\
\hline 39 & - & -99 & 90 & 85 & 39 & 18 & 35 & 19 & 10 & 5 & 18 \\
\hline 38 & - & 98 & 89 & 81 & 38 & 17 & 31 & 16 & 7 & 4 & 17 \\
\hline 37 & - & 97 & 87 & 77 & 37 & 16 & 29 & 13 & 5 & 3 & 16 \\
\hline 36 & - & 96 & 84 & 73 & 36 & 15 & 23 & 11 & 4 & 2 & 15 \\
\hline 35 & 99 & 95 & 81 & 69 & 35 & 14 & 17 & 8 & 3 & 2 & 14 \\
\hline 34 & 98 & 93 & 77 & 65 & 34 & 13 & 15 & 7 & 2 & 1 & 13 \\
\hline 33 & 97 & 90 & 73 & 60 & 33 & 12 & 11 & 6 & 1 & & 12 \\
\hline 32 & 96 & 89 & 69 & 55 & 32 & 11 & 8 & 5 & & & 11 \\
\hline 31 & 95 & 87 & 65 & 50 & 31 & 10 & 6 & 4 & & & 10 \\
\hline 30 & 94 & 84 & 60 & 48 & 30 & 9 & 5 & 3 & & & 9 \\
\hline 29 & 93 & 81 & 55 & 45 & 29 & 8 & 3 & 2 & & & 8 \\
\hline
\end{tabular}


TABLA N $\mathbf{N}^{\circ} 10$

TAE 1 FACTOR CALCULO

PUNTUACIONES PERCENTILES POR GRADO ESCOLAR

\begin{tabular}{|c|c|c|c|c|c|c|c|c|c|c|c|}
\hline \multicolumn{6}{|c|}{ GRADOS ESCOLARES } & \multicolumn{6}{|c|}{ GRADOS ESCOLARES } \\
\hline P.D. & 3ro & 4to & 5 to & 6to & P.D. & P.D. & 3ro & 4to & 5to & 6to & P.D. \\
\hline 30 & - & - & - & - & 30 & 15 & 52 & 35 & 27 & 21 & 15 \\
\hline 29 & - & - & - & - & 29 & 14 & 45 & 27 & 19 & 11 & 14 \\
\hline 28 & - & - & - & 99 & 28 & 13 & 40 & 21 & 13 & 8 & 13 \\
\hline 27 & - & 99 & 99 & 98 & 27 & 12 & 35 & 16 & 10 & 5 & 12 \\
\hline 26 & - & 98 & 97 & 96 & 26 & 11 & 31 & 12 & 7 & 3 & 11 \\
\hline 25 & 99 & 96 & 95 & 93 & 25 & 10 & 27 & 7 & 4 & 2 & 10 \\
\hline 24 & 98 & 94 & 92 & 88 & 24 & 9 & 21 & 5 & 2 & 1 & 9 \\
\hline 23 & 97 & 89 & 85 & 83 & 23 & 8 & 16 & 4 & 1 & & 8 \\
\hline 22 & 96 & 87 & 79 & 77 & 22 & 7 & 12 & 2 & & & 7 \\
\hline 21 & 93 & 81 & 73 & 71 & 21 & 6 & 7 & 1 & & & 6 \\
\hline 20 & 89 & 73 & 69 & 62 & 20 & 5 & 4 & & & & 5 \\
\hline 19 & 84 & 67 & 60 & 50 & 19 & 4 & 2 & & & & 4 \\
\hline 18 & 77 & 57 & 50 & 40 & 18 & 3 & 1 & & & & 3 \\
\hline 17 & 71 & 50 & 43 & 31 & 17 & 2 & & & & & 2 \\
\hline 16 & 62 & 43 & 35 & 23 & 16 & 1 & & & & & 1 \\
\hline
\end{tabular}


TABLA N $\mathbf{N}^{0} 11$

TAE 1 FACTOR CALCULO

PUNTUACIONES PERCENTILES POR GRADO ESCOLAR

\begin{tabular}{|c|c|c|c|c|c|c|c|c|c|c|c|}
\hline \multicolumn{6}{|c|}{ GRADOS ESCOLARES } & \multicolumn{6}{|c|}{ GRADOS ESCOLARES } \\
\hline P.D. & 3ro & 4to & 5 to & 6to & P.D. & P.D. & 3ro & 4 to & 5to & 6to & P.D. \\
\hline 43 & & & & & 43 & 25 & 98 & 87 & 71 & 65 & 25 \\
\hline 42 & & & & & 42 & 24 & 97 & 84 & 65 & 55 & 24 \\
\hline 41 & & & & & 41 & 23 & 96 & 77 & 57 & 50 & 23 \\
\hline 40 & & & & & 40 & 22 & 94 & 71 & 50 & 45 & 22 \\
\hline 39 & & & & & 39 & 21 & 93 & 65 & 48 & 40 & 21 \\
\hline 38 & & & & & 38 & 20 & 89 & 55 & 43 & 31 & 20 \\
\hline 37 & & & & & 37 & 19 & 84 & 50 & 38 & 27 & 19 \\
\hline 36 & & & & & 36 & 18 & 77 & 43 & 33 & 21 & 18 \\
\hline 35 & - & - & - & 99 & 35 & 17 & 69 & 35 & 27 & 17 & 17 \\
\hline 34 & - & - & 99 & 98 & 34 & 16 & 60 & 27 & 23 & 13 & 16 \\
\hline 33 & - & - & 98 & 96 & 33 & 15 & 50 & 21 & 19 & 11 & 15 \\
\hline 32 & - & - & 97 & 94 & 32 & 14 & 48 & 16 & 16 & 7 & 14 \\
\hline 31 & - & 99 & 95 & 93 & 31 & 13 & 43 & 11 & 13 & 5 & 13 \\
\hline 30 & - & 98 & 93 & 89 & 30 & 12 & 38 & 8 & 11 & 4 & 12 \\
\hline 29 & - & 96 & 89 & 85 & 29 & 11 & 33 & 6 & 8 & 2 & 11 \\
\hline 28 & - & 95 & 85 & 79 & 28 & 10 & 29 & 4 & 6 & 1 & 10 \\
\hline 27 & - & 92 & 83 & 73 & 27 & 9 & 25 & 3 & 4 & & 9 \\
\hline 26 & 99 & 89 & 75 & 69 & 26 & 8 & 21 & 2 & 3 & & 8 \\
\hline
\end{tabular}


TABLA N $\mathbf{N}^{\circ} 12$

TAE 1 FACTOR CALCULO PUNTUACIONES PERCENTILES POR GRADO ESCOLAR

\begin{tabular}{|c|c|c|c|c|c|c|c|c|c|c|c|}
\hline \multicolumn{6}{|c|}{ GRADOS ESCOLARES } & \multicolumn{6}{|c|}{ GRADOS ESCOLARES } \\
\hline P.D. & 3ro & 4to & 5to & 6to & P.D. & P.D. & 3ro & 4to & 5to & 6to & P.D. \\
\hline 120 & & & & & 120 & 63 & 79 & 50 & 40 & 27 & 63 \\
\hline 117 & & & & & 117 & 60 & 71 & 48 & 33 & 19 & 60 \\
\hline 114 & & & & & 114 & 57 & 62 & 43 & 29 & 13 & 57 \\
\hline 11 & & & & & 11 & 54 & 50 & 38 & 25 & 8 & 54 \\
\hline 108 & & & & & 108 & 51 & 48 & 29 & 19 & 5 & 51 \\
\hline 105 & & & & & 105 & 48 & 43 & 23 & 15 & 3 & 48 \\
\hline 102 & - & - & - & 99 & 102 & 45 & 38 & 17 & 11 & 2 & 45 \\
\hline 99 & - & - & 99 & 98 & 99 & 42 & 33 & 12 & 7 & 1 & 42 \\
\hline 96 & - & - & 98 & 97 & 96 & 39 & 27 & 8 & 4 & & 39 \\
\hline 93 & - & 99 & 96 & 96 & 93 & 36 & 19 & 6 & 3 & & 36 \\
\hline 90 & - & 98 & 95 & 93 & 90 & 33 & 15 & 4 & 2 & & 33 \\
\hline 87 & 99 & 96 & 93 & 89 & 87 & 30 & 10 & 2 & 1 & & 30 \\
\hline 84 & 98 & 95 & 89 & 83 & 84 & 27 & 7 & 1 & & & 27 \\
\hline 81 & 97 & 93 & 85 & 75 & 81 & 24 & 5 & & & & 24 \\
\hline 78 & 96 & 89 & 79 & 69 & 78 & 21 & 3 & & & & 21 \\
\hline 75 & 95 & 84 & 75 & 60 & 75 & 18 & 2 & & & & 18 \\
\hline 72 & 93 & 77 & 69 & 50 & 72 & 15 & 1 & & & & 15 \\
\hline 69 & 89 & 67 & 60 & 43 & 69 & 12 & & & & & 12 \\
\hline 66 & 85 & 57 & 50 & 33 & 66 & 09 & & & & & 09 \\
\hline
\end{tabular}


TABLA N ${ }^{\circ} 13$

TAE 2 FACTOR VERBAL

PUNTUACIONES PERCENTILES POR GRADO ESCOLAR

\begin{tabular}{|c|c|c|c|c|c|c|c|c|c|c|c|}
\hline \multicolumn{6}{|c|}{ GRADOS ESCOLARES } & \multicolumn{6}{|c|}{ GRADOS ESCOLARES } \\
\hline P.D. & 1ro & 2 do & 3 ro & 4 to & 5 to & P.D. & 1ro & $2 \mathrm{do}$ & 3ro & 4 to & 5to \\
\hline 42 & - & - & - & - & 99 & 24 & 62 & 50 & 35 & 29 & 19 \\
\hline 41 & - & - & - & - & 98 & 23 & 55 & 45 & 29 & 25 & 15 \\
\hline 40 & - & - & - & 99 & 97 & 22 & 50 & 38 & 25 & 19 & 11 \\
\hline 39 & - & - & - & 98 & 95 & 21 & 48 & 33 & 21 & 15 & 7 \\
\hline 38 & - & 99 & 99 & 97 & 92 & 20 & 43 & 27 & 16 & 11 & 5 \\
\hline 37 & - & 98 & 98 & 95 & 87 & 19 & 38 & 23 & 12 & 7 & 4 \\
\hline 36 & - & 98 & 96 & 93 & 83 & 18 & 33 & 19 & 8 & 5 & 2 \\
\hline 35 & - & 96 & 95 & 90 & 79 & 17 & 29 & 15 & 6 & 4 & 1 \\
\hline 34 & - & 95 & 93 & 85 & 73 & 16 & 25 & 11 & 4 & 2 & \\
\hline 33 & 99 & 94 & 90 & 83 & 67 & 15 & 19 & 8 & 3 & 1 & \\
\hline 32 & 98 & 92 & 87 & 79 & 62 & 14 & 13 & 5 & 1 & & \\
\hline 31 & 97 & 89 & 83 & 75 & 55 & 13 & 10 & 4 & & & \\
\hline 30 & 96 & 87 & 75 & 69 & 50 & 12 & 6 & 2 & & & \\
\hline 29 & 93 & 83 & 69 & 60 & 43 & 11 & 5 & 1 & & & \\
\hline 28 & 89 & 77 & 60 & 50 & 38 & 10 & 3 & & & & \\
\hline 27 & 87 & 69 & 50 & 45 & 33 & 9 & 12 & & & & \\
\hline 26 & 79 & 62 & 45 & 38 & 29 & 8 & 1 & & & & \\
\hline 25 & 71 & 55 & 40 & 33 & 25 & & & & & & \\
\hline
\end{tabular}


TABLA No 14

TAE 2 FACTOR RAZONAMIENTO

PUNTUACIONES PERCENTILES POR GRADO ESCOLAR

\begin{tabular}{|c|c|c|c|c|c|c|c|c|c|c|c|}
\hline \multicolumn{6}{|c|}{ GRADOS ESCOLARES } & \multicolumn{6}{|c|}{ GRADOS ESCOLARES } \\
\hline P.D. & 1ro & $2 \mathrm{do}$ & 3 ro & 4 to & 5 to & P.D. & 1ro & $2 \mathrm{do}$ & 3 ro & 4to & 5to \\
\hline 30 & - & - & - & 98 & 96 & 15 & 75 & 50 & 38 & 27 & 16 \\
\hline 29 & - & - & 99 & 96 & 94 & 14 & 67 & 45 & 31 & 21 & 11 \\
\hline 28 & - & - & 98 & 94 & 92 & 13 & 60 & 40 & 25 & 16 & 7 \\
\hline 27 & - & - & 97 & 93 & 88 & 12 & 50 & 33 & 19 & 12 & 5 \\
\hline 26 & - & - & 95 & 90 & 84 & 11 & 40 & 27 & 15 & 8 & 3 \\
\hline 25 & - & 99 & 93 & 87 & 77 & 10 & 33 & 21 & 11 & 6 & 3 \\
\hline 24 & 99 & 98 & 90 & 83 & 71 & 9 & 27 & 16 & 7 & 4 & 1 \\
\hline 23 & 98 & 96 & 87 & 77 & 65 & 8 & 19 & 12 & 5 & 2 & \\
\hline 22 & 96 & 95 & 83 & 71 & 57 & 7 & 13 & 8 & 3 & 1 & \\
\hline 21 & 95 & 93 & 77 & 65 & 50 & 6 & 8 & 6 & 2 & & \\
\hline 20 & 93 & 89 & 71 & 55 & 45 & 5 & 5 & 4 & 1 & & \\
\hline 19 & 89 & 84 & 65 & 50 & 38 & 4 & 3 & & & & \\
\hline 18 & 85 & 77 & 57 & 45 & 31 & 3 & & & & & \\
\hline 17 & 83 & 69 & 50 & 40 & 25 & 2 & & & & & \\
\hline 16 & 79 & 60 & 45 & 33 & 21 & 1 & & & & & \\
\hline
\end{tabular}


TABLA N ${ }^{\circ} 15$

TAE 2 FACTOR CALCULO

PUNTUACIONES PERCENTILES POR GRADO ESCOLAR

\begin{tabular}{|c|c|c|c|c|c|c|c|c|c|c|c|}
\hline \multicolumn{6}{|c|}{ GRADOS ESCOLARES } & \multicolumn{6}{|c|}{ GRADOS ESCOLARES } \\
\hline P.D. & 1ro & $2 \mathrm{do}$ & 3 ro & 4 to & 5 to & P.D. & 1ro & $2 \mathrm{do}$ & 3 ro & 4to & 5to \\
\hline 30 & - & - & - & 99 & 97 & 15 & 75 & 55 & 38 & 31 & 17 \\
\hline 29 & - & - & 99 & 98 & 95 & 14 & 67 & 50 & 31 & 25 & 13 \\
\hline 28 & - & - & 98 & 97 & 93 & 13 & 55 & 45 & 25 & 19 & 10 \\
\hline 27 & - & - & 97 & 95 & 89 & 12 & 50 & 38 & 19 & 15 & 7 \\
\hline 26 & - & - & 95 & 93 & 85 & 11 & 45 & 31 & 15 & 11 & 5 \\
\hline 25 & - & 99 & 93 & 90 & 79 & 10 & 38 & 25 & 11 & 7 & 3 \\
\hline 24 & - & 98 & 90 & 87 & 73 & 9 & 31 & 19 & 7 & 5 & 2 \\
\hline 23 & 99 & 97 & 87 & 83 & 65 & 8 & 27 & 15 & 5 & 3 & 1 \\
\hline 22 & 98 & 95 & 83 & 77 & 57 & 7 & 21 & 11 & 4 & 2 & \\
\hline 21 & 97 & 93 & 77 & 71 & 50 & 6 & 16 & 7 & 2 & 1 & \\
\hline 20 & 96 & 88 & 71 & 65 & 45 & 5 & 12 & 5 & 1 & & \\
\hline 19 & 93 & 83 & 65 & 57 & 40 & 4 & 8 & 4 & & & \\
\hline 18 & 90 & 75 & 57 & 50 & 35 & 3 & 6 & 2 & & & \\
\hline 17 & 87 & 69 & 50 & 45 & 29 & 2 & 4 & 1 & & & \\
\hline 16 & 83 & 62 & 45 & 38 & 23 & 1 & 3 & & & & \\
\hline
\end{tabular}


TABLA N ${ }^{\circ} 16$

TAE 2 PUNTUACION TOTAL

PUNTUACIONES PERCENTILES POR GRADO ESCOLAR

\begin{tabular}{|c|c|c|c|c|c|c|c|c|c|c|c|}
\hline \multicolumn{6}{|c|}{ GRADOS ESCOLARES } & \multicolumn{6}{|c|}{ GRADOS ESCOLARES } \\
\hline P.D. & 1ro & 2 do & 3 ro & 4 to & 5 to & P.D. & 1ro & $2 \mathrm{do}$ & 3 ro & 4to & 5 to \\
\hline 103 & - & - & - & 99 & 99 & 55 & 71 & 50 & 31 & 25 & 11 \\
\hline 100 & - & - & - & 98 & 99 & 51 & 65 & 45 & 25 & 19 & 7 \\
\hline 97 & - & - & - & 97 & 97 & 48 & 57 & 38 & 19 & 115 & 5 \\
\hline 95 & - & - & 99 & 95 & 95 & 45 & 52 & 31 & 13 & 11 & 4 \\
\hline 91 & - & - & 98 & 93 & 93 & 42 & 43 & 25 & 8 & 7 & 2 \\
\hline 88 & - & - & 97 & 90 & 88 & 39 & 35 & 19 & 7 & 5 & 1 \\
\hline 85 & - & - & 95 & 87 & 83 & 36 & 27 & 15 & 5 & 3 & \\
\hline 82 & - & 99 & 92 & 83 & 75 & 33 & 19 & 11 & 3 & 2 & \\
\hline 79 & 99 & 97 & 87 & 77 & 67 & 30 & 13 & 7 & 2 & & \\
\hline 76 & 98 & 95 & 81 & 71 & 57 & 27 & 8 & 5 & 1 & & \\
\hline 73 & 96 & 93 & 75 & 65 & 48 & 24 & 5 & 3 & & & \\
\hline 70 & 94 & 88 & 67 & 57 & 40 & 21 & 3 & 2 & & & \\
\hline 67 & 90 & 83 & 60 & 50 & 33 & 18 & 2 & 1 & & & \\
\hline 64 & 87 & 75 & 52 & 45 & 27 & 15 & 1 & & & & \\
\hline 61 & 83 & 67 & 45 & 38 & 19 & 12 & & & & & \\
\hline 58 & 77 & 57 & 38 & 31 & 15 & & & & & & \\
\hline
\end{tabular}




\section{REFERENCIAS}

ANASTASI, ANA "Tests Psicológicos" Edit. Aguilar, Madrid, 1979.

ALLEN VETA W "Introducción a la Medición Psicológica" Monterrey, Brooks Cole, USA. 1989.

ADAMS SACHS "Medición y Evaluación" Edit. Herder. Madrid.

GONZALES. G. "Revisión de Confiabilidad, Validez del TAE 1" Tesis, para Titulo Profesional. UNMSM,1991.

GUILLFORD, J. "Métodos Psicométricos" Edit, Prentice-Hall.

HORROCKS. J. "Psicología de la Adolescencia" Edit. Trillas.

LEWIS. D. "Métodos cuantitativos en Psicología" Ed.Prentice Hall, 1987.

LINDERMAN. R. "Tratado de Medición Educacional" Edit. Paidós

NUNNALL V, J. C. "Teoría Psicométrica" Edit. Trillas, 1987.

NOEL RUTH. "Standarización del TAE 1 en el Callao" Tesis para Titulo Profesional. UNMSM.1992.

OETTING, W. "Prácticas de Psicometría" Edit.Trillas.

PASQUASY, R. "Las aptitudes y su Medida" Edit. Morava, Madrid

RUIZ, CESAR Ejercicios de Complemenaión: Aplicación Práctica de la Medición

Psicológica, Separata. Dpto. Impresiones, Psicología, UNMSM. 1993.

THURSTONE, L. "La medición de la Inteligencia, la aptitud y el interés" Edit. Paidós, BS.AS.

TVLER, L. "Pruebas y Medición en Psicología" Edit. Prentice Hall, 1982.

YELA, MARIANO "Psicología de las aptitudes" Edit, Gredos. 